\title{
Polarizability relaxation in water/ethanol mixtures
}

\author{
Ary de Oliveira Cavalcante ${ }^{\mathrm{a}, *}$, Riccardo Chelli ${ }^{\mathrm{b}}$ \\ a Laboratório de Espectroscopia e Dinâmica Molecular Departamento de Química, Universidade Federal do Amazonas, Av. Rodrigo Otávio, 6200, Coroado, Manaus, AM, Brazil \\ b Dipartimento di Chimica, Università di Firenze, Via della Lastruccia 3, I-50019, Sesto Fiorentino, Italy
}

\section{A R T I C L E I N F O}

\section{Article history:}

Received 6 July 2020

Received in revised form 25 January 2021

Accepted 1 March 2021

Available online 03 March 2021

\section{Keywords:}

Low-frequency Raman spectroscopy

Polarizability relaxation

Molecular dynamics

Binary mixtures

\begin{abstract}
A B S T R A C T
Molecular dynamics simulations are used to investigate structural and dynamical properties of liquid mixtures of water and ethanol over the entire range of compositions from neat liquid water to neat liquid ethanol. Particular emphasis is given to the time relaxation of the anisotropic collective polarizability and the low-frequency part of the depolarized Raman spectra. While the dynamics was carried out using simulations with standard force fields for water and ethanol, the post-analysis was performed employing the chemical potential equalization (CPE) method. For comparison purposes, the CPE results are compared to those obtained with the traditional interaction-induced model. Both methods are able to capture the basic shape and position of the Raman bands. Polarizability response in time and frequency domains is disentangled in permanent, interaction-induced and induced-permanent (cross) terms. Our findings suggest that the polarizability relaxation of water is more sensitive to environmental fluctuations. Once water signal remains covered by the ethanol contribution even in waterrich concentrations, some water polarizability features were unveiled and correlated with $\mathrm{H}$-bond dynamics. The permanent term, representative of the reorientational dynamics, captures the short-time oscillations and responds for a part of the total relaxation, while the long-range interaction-induced contribution is responsible for great part of the total polarizability relaxation.
\end{abstract}

(C) 2021 Elsevier B.V. All rights reserved.

\section{Introduction}

Binary mixtures of molecular liquids are traditional subjects of investigation in the field of Molecular Dynamics (MD) simulations [1-5]. Textbooks of physical chemistry define the ideal solutions as those whose components are randomly mixed at the molecular level. However, small chemical differences between molecules may give rise to deviations from the ideal behavior, so that local heterogeneities as well as small molecular level concentration deviations may take place. These systems were subject to intense investigation and their non-ideal behavior could provide a proper key to study fundamental aspects of the intermolecular interactions and also to address issues useful for the development of materials, industry, and processes.

It is widely accepted that the properties of a great variety of liquids, from simple solvents up to very complex solutions of macromolecules typical of living organisms, are fundamentally determined, or strongly influenced, by the structure and dynamics of H-bonds. In the present case, in spite of the simplicity of the molecular species involved, there is a delicate and complex balance between several possible interactions, such as H-bonding, dipole-dipole, or induced-dipole interactions. As a consequence, deviations from the ideal behavior are expected and

\footnotetext{
* Corresponding author.

E-mail address: arycavalcante@ufam.edu.br (A. de Oliveira Cavalcante).
}

there is an interest in the proper characterization of the so-called nonideal behavior of binary mixtures.

A typical molecular level non-ideal phenomenon concerning alcoholic aqueous solutions is the counterintuitive and controversial apparent ability of the water molecules to keep its H-bonded nearestneighbors structure, seen in the pure liquid, with increasing amounts of alcohol. The behavior of the sound speed in water-ethanol binary mixtures [2] typically exhibits an unexpected maximum at the specific water molar fraction of $\chi_{w} \sim 0.85$. According to thermodynamics, at this concentration, there is a minimum in the excess enthalpy [6]. Both of those experimental results are currently explained by the persistence of the H-bond network structure in pure liquid water or, in other words, to the reinforcement of the water-water H-bond network in water-rich mixtures. A very similar enhancement of the interactions was also seen in ethanol rich mixtures [7], leading to a reduction in entropy and a negative enthalpy of mixing, consistent with existing thermodynamic data.

Also in water-rich alcoholic mixtures, under high pressures and/or low temperatures [8,9], a complex and collective structural arrangement takes place. As a possible consequence of two main trends, namely, the minimization of the hydrophobic intermolecular interactions and the maximization/enhancement of the water-water interactions (H-bonds), maybe the formation of clathrate hydrates [10]. Also known as hydrophobic hydration, these clathrate hydrate structures are characterized by ethanol molecules surrounded by a cage formed 
by H-bonded water molecules [11] with long-lived ( $10 \mathrm{ps)} \mathrm{host-guest}$ in the hydrate phases while maintaining the mentioned cage structure.

Despite the apparent simplicity of the water-ethanol solutions, we have a clear and broad landscape in which the alcoholic-water mixture's phenomenology is spread out. Usually, the formation/breaking of $\mathrm{H}$-bonds, as well as its consequences on the local structural order, are often considered as one of the main reasons for the complex behavior of the thermodynamic properties like the excess enthalpy of mixing or the heat capacity [12]. In this respect, MD simulations emerge as a reliable tool for the proper investigation over the entire range of composition, denoted in this work as the water molar fraction. In this sense, previous MD simulations have been done elucidating the details about the short-range structure [5], thermodynamics, diffusion properties, and viscosity [3].

Over the last decades, several attempts have been made to study polarizability trends in frequency [12-15] and time domains [16,17] from computer simulations. The main differences in the calculation of the polarizability consist of how the series expansion around the unperturbed gas-phase value accounts for the condensed phase interactions (usually, a first order dipole-induced dipole approximation is employed). Refinements on the both description of the intermolecular interactions [18] as well as the intramolecular polarizability if found for a great diversity of systems like pure molecular liquids [2-8] and binary mixtures [9-11]. Alternatively, a more complex expansion with terms embedded with specific quantum meaning have been proposed for the polarizable ionic species in molten salts $[19,20]$, where the electrostatic interactions are responsible for a peculiar compression of the electron cloud. We highlight also the full ab initio MD based polarizability computations $[21,22]$. In this sense, CPE polarizability calculations is placed as an alternative attempt that combines both the refinement on molecular polarizability description as well as the accuracy of its interactions.

The aim of the present study is to unveil the polarizability dynamics in water/ethanol mixtures along the entire range of composition and to investigate its possible connections to relevant structural features of the system. Particular attention is reserved to H-bonding dynamical features, which are revisited and placed in the current context. The behavior of the polarizability in time and frequency domains has been investigated using a hybrid approach according to which, while dynamics is performed using a well established non polarizable force field, the dependence of the electronic density on the chemical environment is accounted for using an empirical method based on the chemical potential equalization, the so-called CPE model $[23,24]$. We point out that, despite the apparent simplicity of the system under study, water and ethanol and their mixtures actually carry a wide complexity, especially related to the subtle balancing of the strong H-bonding interactions, which have a significant impact in the optical properties. This prompt us with an archetypical and challenging task, properly developing, testing and applying the CPE model to a relevant problem.

This paper is organized as follows. In Section 2, we present a description of the computational details and models used in this study and provide the protocol for the parameterization of the CPE model. Results and discussion are reported in Section 3. Concluding remarks are given in Section 4.

\section{Theory and methods}

\subsection{Chemical potential equalization method}

The chemical potential equalization (CPE) method [12,25-33] is based on the fundamental idea that the chemical potential in a molecule must be equal through the whole molecule at the equilibrium [34]. The concept of chemical potential within a molecule is similar to that of chemical potential into solution. While the latter is related to the equilibrium of different chemical species into a mixture, the former deals with the equilibrium of atomic charges, or better, of electron density, within a molecule. Thus, the atomic chemical potentials give a criterion for the dynamics of a net charge inward or outward the atoms. Electron density flow takes place from regions with high chemical potential to regions with low chemical potential, until reaching the chemical potential balance through the whole molecule. From the standpoint of a point-charge distribution, such a criterion establishes that the negative charges flow from sites with high chemical potential to sites with low chemical potential (vice versa for the case of positive charges), maintaining the overall charge balance. The chemical potential $\mu_{i}$ of an atom $i$ in a molecule equals the negative of the atomic electronegativity $\chi_{i}$ and is given by [35]

$\mu_{i}=-\chi_{i}=-\frac{\partial E}{\partial Q_{i}}$

where $Q_{i}$ is the atomic net charge on the atom $i$ and $E$ is the molecular energy. At equilibrium, the chemical potential should be equalized everywhere in the molecule $[28,29,35]$, so that

$\chi_{1}=\chi_{2}=\chi_{3}=\cdots=\chi_{M}$

where $M$ is the number of atoms in the molecule. By expanding the energy to the second order in the atomic charges, we obtain $[27,28,30]$

$E=E_{0}+\sum_{j} \chi_{j}^{0} Q_{j}+\frac{1}{2} \sum_{j} \eta_{j}^{0} Q_{j}^{2}+\frac{1}{2} \sum_{j} \sum_{k \neq j} \frac{Q_{j} Q_{k}}{R_{j k}}$

where $\chi_{j}^{0}$ and $\eta_{j}^{0}$ are the reference atomic electronegativity and hardness, respectively, $E_{0}$ is a coordinate-dependent term modeled through the stretching, bending, and torsional potentials, and $R_{j k}$ is the distance between the atoms $j$ and $k$. Note that the last term into Eq. 3 corresponds to the Coulomb energy (for the sake of simplicity, we have omitted the coefficient dependent on the dielectric constant of vacuum). In more sophisticated CPE approaches, the Coulomb interaction into Eq. 3 is modeled through a screening factor including form factors for the charge distribution that can be of Slater $[27,28,30]$ or Gaussian [32] type. Here, we use the simplest approach, considering the atomic charges as point charges. As pointed out in several studies [27,28,31,36,37], $\chi_{j}^{0}$ and $\eta_{j}^{0}$ do not refer to isolated atoms, but depend on the nuclear configuration. Within the assumption of small oscillations around an average nuclear configuration, these quantities are taken to be constant parameters fitted to molecular electronic properties [31,38-43]. Combining Eqs. 1 and 3 allows us to recover the expression of the atomic electronegativity in terms of atomic charges and nuclear configuration.

$\chi_{i}=\chi_{i}^{0}+\eta_{i}^{0} Q_{i}+\sum_{j \neq i} \frac{Q_{j}}{R_{i j}}$

The $M-1$ equations reported in Eq. 2, after substituting Eq. 4, together with the electroneutrality condition $\sum_{i=1}^{M} Q_{i}=0$, can be cast in a system of $M$ linear equations in the $Q_{1}, Q_{2}, \ldots, Q_{M}$ variables. Such equations can be reported in a matrix form as follows

$\mathbf{J} \mathbf{Q}=\Delta \mathbf{K}$

where $\Delta \mathbf{K}=\left(0, \chi_{1}^{0}-\chi_{2}^{0}, \ldots, \chi_{1}^{0}-\chi_{M}^{0}\right), \mathbf{Q}=\left(Q_{1}, Q_{2}, \ldots, Q_{M}\right)$ and the generic matrix element $J_{i j}$ is given by

$J_{i j}=\delta_{1 i}+G_{i j}-G_{1 j}$

with

$G_{i j}=\delta_{i j} \eta_{i}^{0}+\left(1-\delta_{i j}\right) / R_{i j}$

where $\delta_{i j}$ is the Kronecker delta. The tendency to charge transfer between atoms is also regulated by the local hardness $\eta_{i}^{0}$ : large hardness inhibits release or acceptance of charge. The equilibrium charge 
distribution depends on the instantaneous nuclear configuration through the Coulomb interaction energy and on the value of the local external field, which affects in different ways the electronegativities of the atoms in the molecule (see Eq. 4).

Many electronic properties can be calculated using CPE. The dipole moment $\mathbf{m}=\sum_{i=1}^{M} Q_{i} \mathbf{r}_{i}$ of the isolated molecule is obtained solving Eq. 5 for $\mathbf{Q}$. In order to find the molecular polarizability, we apply a constant electric field $\varepsilon$ in the $\gamma$ direction and recalculate the charge distribution adding to the energy of Eq. 3 the term due to the field, i.e., $-\varepsilon \sum_{i=1}^{M} Q_{i} r_{\gamma i}$, with $r_{\gamma i}$ being the $\gamma$ th Cartesian coordinate of atom $i$. Equalizing the electronegativities and imposing electroneutrality, we find that the new charges obey the equation

$\mathbf{J}(\mathbf{Q}+\delta \mathbf{Q})=\Delta \mathbf{K}+\varepsilon \Delta \mathbf{r}_{\gamma}$

where $\mathbf{J}$ is the matrix defined in Eq. 6, $\mathbf{Q}$ is the unperturbed charge array vector, $\delta \mathbf{Q}$ is the vector representing the atomic-charge change induced by the electric field and $\Delta \mathbf{r}_{\gamma}=\left(0, r_{\gamma 2}-r_{\gamma 1}, \ldots, r_{\gamma M}-r_{\gamma 1}\right)$. Inserting Eq. 5 into Eq. 8 and solving the resulting equation for $\delta \mathbf{Q}$ we can recover the induced dipole moment along the $\beta$ direction due to the electric field in the $\gamma$ direction as

$m_{\beta}=\varepsilon \sum_{i=1}^{M} \sum_{j=1}^{M}\left[\mathbf{J}^{-1}\right]_{i j} r_{\beta i} \Delta r_{\gamma j}$

The molecular polarizability tensor element is thus given by the matrix equation

$\alpha_{\gamma \beta}=\mathbf{r}_{\beta} \mathbf{J}^{-1} \Delta \mathbf{r}_{\gamma}$

The polarizability tensor yields the global response of the molecule to the applied external field.

In the liquid state, the molecular electron density is perturbed by the additional potential due to the surrounding molecules. For a given nuclear configuration of the liquid, the CPE energy of a generic molecule $l$ is therefore given by

$E_{l}=E_{0 l}+\sum_{i=1}^{M} \mathrm{~V}_{i l} Q_{i l}$

where $E_{0 l}$ is the gas-phase energy of Eq. 3 and $V_{i l}$ is the electrostatic potential due to all other molecules acting on the atom $i$ of the $l$ th molecule. This potential depends linearly on the atomic charges of all other molecules. The charges on the molecule $l$ are determined by solving the $L$ coupled equations

$\mathbf{J}_{l} \mathbf{Q}_{l}=\Delta \mathbf{K}+\Delta \mathbf{V}_{l}$

where $\mathbf{J}_{l}$ is the hardness matrix defined in Eq. $6, \Delta \mathbf{K}$ is the differential electronegativity vector of Eq. 5 and $L$ is the number of molecules in the sample. In Eq. 12, we have equalized the derivative with respect to the atomic charges, used the condition of electroneutrality, and defined $\Delta \mathbf{V}_{l}=\left(0, V_{1 l}-V_{2 l}, \ldots, V_{1 l}-V_{M l}\right)$. Eq. 12 makes clear that the local electrostatic potential due to the surrounding molecules adds up to the electronegativity of the atoms. A negative electrostatic potential at the atom decreases its electronegativity, while a positive potential increases it. Since the potential $V_{i l}$ depends linearly on the atomic charges, we may set up a system of $L \times M$ linear equations to be solved for the $L \times M$ atomic charges.

\subsection{Polarizability time correlation functions and anisotropic Raman spectra}

The depolarized Raman spectra are related to the relaxation of the polarizability anisotropy of the system and can be described by the Fourier transform of the time correlation function (TCF) of an off-diagonal component $\Pi_{x z}$ of the collective polarizability tensor $\Pi$ [44-47], that simply corresponds to the sum of the molecular polarizabilities. The TCF of $\Pi_{x z}$ is properly defined as

$\Psi_{x y}(t)=\frac{\left\langle\Pi_{x y}(t) \Pi_{x y}(0)\right\rangle}{\frac{1}{15} N\left\langle\gamma^{2}\right\rangle}$

where the angular brackets denote ensemble averages, $N$ is the number of molecules and $\gamma^{2}$ is the square of the polarizability anisotropy of an isolated molecule

$\gamma^{2}=\frac{1}{2}\left[\left(\alpha_{x x}-\alpha_{y y}\right)^{2}+\left(\alpha_{x x}-\alpha_{z z}\right)^{2}+\left(\alpha_{y y}-\alpha_{z z}\right)^{2}+6\left(\alpha_{x y}^{2}+\alpha_{x z}^{2}+\alpha_{y z}^{2}\right)\right]$

In a quite simple picture, the polarizability tensor $\Pi$ in condensed phases can be split into two parts. The so-called permanent or molecular part, $\Pi^{M}$, that depends only on the molecular orientational coordinates in the lab frame (and conformations of flexible molecules), and the interaction-induced part, $\Pi^{I}$, that arises from the interactions of the molecular-induced dipole moments and accounts for the intermolecular interactions. In other words, the interaction-induced part depends on all degrees of freedom, including the translational ones, and essentially is the source of the bands seen in the low frequency part of the vibrational Raman spectra. Often, the polarizability expansion is truncated at the first-order dipole-induced-term as follows.

$\Pi=\Pi^{M}+\Pi^{I}$

where $\Pi^{M}$ is the sum of the orientational polarizabilities $\boldsymbol{\alpha}_{i}$ of all $N$ molecules,

$\Pi^{M}=\sum_{i=1}^{N} \boldsymbol{\alpha}_{i}$

The polarizability term $\Pi^{I}$ arises from the interactions between molecular induced dipoles and depends on orientational, conformational and translational degrees of freedom (see Ref. 37 and references therein).

$\Pi^{I} \cong \sum_{i=1}^{N} \sum_{j \neq i}^{N} \boldsymbol{\alpha}_{i} \cdot \mathbf{T}\left(\boldsymbol{r}_{i j}\right) \cdot \boldsymbol{\alpha}_{j}$

where $\mathbf{T}\left(\boldsymbol{r}_{i j}\right)$ is the dipole tensor which depends on the interatomic distance vector $\boldsymbol{r}_{i j}$

$\mathbf{T}(\boldsymbol{r})=\frac{3 \widehat{\mathbf{r}} \widehat{\boldsymbol{r}}-\mathbf{1}}{4 \pi \varepsilon_{0} r^{3}}$

$\widehat{\boldsymbol{r}}=\boldsymbol{r} / r$ being a unit vector along $\boldsymbol{r}$ and $\mathbf{1}$ a unit tensor. Eq. 15 implies that the time relaxation of the anisotropic polarizability, $\Psi(t)$, is a result of three components,

$\Psi(t)=\Psi^{M M}(t)+\Psi^{M I}(t)+\Psi^{I I}(t)$

where

$\Psi^{M M}(t)=\frac{15}{N\left\langle\gamma^{2}\right\rangle}\left\langle\Pi_{x z}^{M}(t) \Pi_{x z}^{M}(0)\right\rangle$

$\Psi^{M I}(t)=\frac{15}{N\left\langle\gamma^{2}\right\rangle}\left(\left\langle\Pi_{x z}^{M}(t) \Pi_{x z}^{I}(0)\right\rangle+\left\langle\Pi_{x z}^{I}(t) \Pi_{x z}^{M}(0)\right\rangle\right)$

$\Psi^{I I}(t)=\frac{15}{N\left\langle\gamma^{2}\right\rangle}\left\langle\Pi_{x z}^{I}(t) \Pi_{x z}^{I}(0)\right\rangle$

Given the splitting of $\Psi(t)$ into three components (Eq. 19), we may also express the Raman spectrum as a sum of three contributions, arising from the Fourier transforms of $\Psi^{M M}(t), \Psi^{M I}(t)$ and $\Psi^{I I}(t)$. As a matter 
of fact, such a splitting does not have a direct experimental counterpart and, until now, the extent of participation of each mechanism to the overall relaxation of the anisotropic polarizability emerges as theoretical information.

\subsection{Molecular dynamics simulations}

The optical properties reported in this study and detailed in Section 2.B, have been computed using the CPE approach as described in Section 2.A. However, it is worthwhile to point out that the system configurations employed to determine such properties have been achieved from standard MD simulations, i.e. using a non-polarizable force field (see below for details). The reason of this choice is twice. First, the implementation of a polarizable force field for addressing the dynamics of a system would require a very complicated parameterization, because repulsion and van der Waals interactions should be considered together with the usual CPE parameters. In analogy with the CPE parameters, such additional Lennard-Jones like parameters should be configuration-dependent rather than constant as in standard simulations. In this respect, we note that the theoretical developments made till today do not provide a solid background for such a type of approach. The second issue, is related to the solution of the equations of motion. The procedure would require heavy calculations because, at each simulation step, a system of many linear equations (one for each polarizable atom/site) should be solved to update the value of the charges on the polarizable atoms/sites.

As stated above, the MD trajectories were generated using a standard force field including the Lennard-Jones potential for short-range intermolecular repulsions and dispersion interactions together with a Coulomb electrostatic potential between point charges located on atomic sites. To model the water molecule, we used the 3-sites singlepoint charge (the so-called SPC) potential model [48]. The full-atom AMBER-like ff99sb force field [49] in combination with atomic charges computed through a RESP fit [50] at the HF/6-31G* level of theory have been used to model the ethanol molecule. The obtained atomic charges as well as the AMBER atom-types assigned to the atoms of the ethanol molecule are reported in Table 1.

Eleven simulations of water/ethanol mixtures at different concentration ratio, including neat water and neat ethanol, were performed. In all simulations, we kept fixed the number of total molecules to 250, while varying the relative amount of ethanol into water. In Table 2 , we report the composition of the mixtures.

A cubic simulation box was used with periodic boundary conditions. Equilibration was obtained with constant-pressure constanttemperature (NPT) simulations, setting the pressure to $0.1 \mathrm{MPa}$ and the temperature to $298 \mathrm{~K}$. The Constant pressure is enforced isotropically using a modification of the Parrinello-Rahman Lagrangian [51] while temperature control is achieved through a Nosé-Hoover thermostat $[52,53]$. The electrostatic interactions are accounted for by using the smooth particle mesh Ewald method [54] with a convergence parameter of $0.43 \AA^{-1}$. The grid spacing along each direction of the space is about $0.75 \AA$, and a fourth-order B-spline interpolation is used for evaluating the gridded charge array. The equations of motion are

Table 1

Atomic charges (a.u.) and AMBER atom-types used for the ethanol molecule.

\begin{tabular}{lll}
\hline Atom & Charge & AMBER atom-type \\
\hline Methyl C & -0.2586 & CT: sp3 aliphatic C \\
Methylene C & 0.3215 & CT: sp3 aliphatic C \\
Methyl H & 0.0761 & HC: aliphatic H bonded to C \\
& & without electron withdrawing group \\
Methylene H & -0.0286 & H1: aliphatic H bonded to C \\
& & with 1 electron withdrawing group \\
O & -0.6137 & OH: O in hydroxyl group \\
Hydroxy H & 0.3797 & HO: H in hydroxyl group \\
\hline
\end{tabular}

Table 2

Column 1: number of water molecules in the sample. Column 2: number of ethanol molecules in the sample. Column 3: molar fraction of water molecules in the sample. Column 4: calculated density (in $\mathrm{g} \mathrm{cm}^{-3}$ ) in constant-pressure constant-temperature simulations. Column 5: experimental density (Table 3-110 (page 3-89) "Perry's Chemical engineers 'Handbook" 6 h Edition).

\begin{tabular}{lllll}
\hline n. $\mathrm{H}_{2} \mathrm{O}$ & n. EtOH & $\chi_{w}$ & $\rho_{\text {calc }}$ & $\rho_{\text {exp }}$ \\
\hline 250 & 0 & 1.0 & 0.976 & 0.997 \\
225 & 25 & 0.9 & 0.928 & 0.962 \\
200 & 50 & 0.8 & 0.885 & 0.933 \\
175 & 75 & 0.7 & 0.851 & 0.905 \\
150 & 100 & 0.6 & 0.826 & 0.880 \\
125 & 125 & 0.5 & 0.805 & 0.858 \\
100 & 150 & 0.4 & 0.788 & 0.841 \\
75 & 175 & 0.3 & 0.744 & 0.825 \\
50 & 200 & 0.2 & 0.761 & 0.811 \\
25 & 225 & 0.1 & 0.750 & 0.797 \\
0 & 250 & 0.0 & 0.739 & 0.785 \\
\hline
\end{tabular}

integrated using a multiple time-step r-RESPA scheme [55], with the greatest time-step equal to $9 \mathrm{fs}$. The cutoff for Lennard-Jones and direct lattice electrostatic interactions is $12 \AA$. Constraints are applied to $\mathrm{CH}$ and $\mathrm{OH}$ covalent bonds using the SHAKE procedure [56]. Simulations were performed with the ORAC program [57], while quantumchemical calculations were performed with the Gaussian 09 program [58]. After the NPT equilibration run, a further constant-volume constant-energy (NVE) equilibration was performed using the average box volume obtained in the NPT run and scaling at regular time steps ( $1 \mathrm{ps}$ ) the temperature to $298 \mathrm{~K}$. The average density of the mixtures obtained in the NPT simulations is compared to the experimental data in Table 2. Finally, a NVE production run lasting 0.9 ns was performed saving the atomic coordinates every $9 \mathrm{f}$. for the post-analysis.

\subsection{Calibration of the CPE parameters}

The analysis presented in this study is essentially based on the system polarizability. This electronic property, as well as the atomic charges, was computed using the CPE approach over the system dynamics produced by standard MD simulations as described in Section 2.C. The CPE parameters were calibrated on quantum-chemical calculations at the MP2 level of theory, using a $6-311 G^{*}$ basis set. Specifically, they were determined by fitting the Mulliken charges and the polarizability tensors of the water and ethanol molecules with and without the application of an external electric field. For the simplest 3-site water model, CPE parameters are reported by various authors $[59,60]$, limiting the atomic charges to fluctuate in the molecular plane. A more sophisticated five-site water model is known in the literature [61] and here it is used to deal with the out-of-plane polarization, exploiting two additional equivalent polarizable $\mathrm{CPE}$ sites close to the $\mathrm{O}$ site (keeping the similarity to the lone pairs of the oxygen). These lone pairs (LP) sites are arranged in symmetrically equivalent positions on the vertical plane of water bisecting the H-O-H angle with a LP-O-LP angle of 109.4 degrees. For ethanol, the arrangement of the two LP sites with respect to the $\mathrm{COH}$ group is similar to water. The O-LP distance, that uniquely defines the position of the LP sites, was allowed to vary in the fit. The other fitting parameters are the electronegativities and the hardnesses of all molecular sites. Such sites are the $\mathrm{O}$ and $\mathrm{H}$ atoms together with the LP sites for water, while for ethanol they are the $\mathrm{CH}_{3}$, and $\mathrm{CH}_{2}$ united-atom groups together with the $\mathrm{O}, \mathrm{H}$ and LP sites. In the case of ethanol, we decided to employ a united-atom CPE model to speed up the calculation, especially in the view of applications to large samples. In order to account for the electronic density located on the lone pairs, the Mulliken charge on the $\mathrm{O}$ atom has been distributed among $\mathrm{O}$ and LP sites, so that $10 \%$ of the total Mulliken charge is assigned to each LP site. The amount of charge in each LP site agrees with the typical average charge value employed in previous 5-sites water models [61-63]. The dipole 
moments of the water and ethanol molecules arising from this redistribution of the oxygen Mulliken charge are about 5\% smaller than the ab initio counterparts. The simplex algorithm [64] was used for the optimization. The parametrization procedure is the following.

1. The CPE parameters were initially restrained to typical ranges used in the literature $[24,65,66]$. With such restraints in place, the fitting parameters were allowed to vary in order to reproduce the gas-phase Mulliken charges distributed in the molecular sites as explained above. The O-LP distance was left fixed at this step.

2. Starting from the fitting parameters obtained in step 1 , a fitting run was performed to reproduce the in-plane polarizability tensor elements.

3. Starting from the fitting parameters obtained in step 2, a fitting run was performed to reproduce the out-of-plane polarizability tensor elements. In this run, the O-LP distance was considered as a tunable parameter, since it affects directly the out-of-plane polarizability tensor elements.

4. The parameters obtained in step 3 were then used as initial parameters in a further fitting run leaving fixed the O-LP distance. In this step, the fitted quantities are the Mulliken charges and the polarizability tensor elements computed quantum-mechanically for 20 configurations of a molecule surrounded by a point charge displaced randomly at a few $\AA$ far from the center of mass.

5. A check of the fitting parameters was then performed by calculating the average dipole moment of water and ethanol for several snapshots of pure water and pure ethanol taken from the MD simulations. If the estimates are consistent with the experimental values [67] then the procedure is stopped. Otherwise, the parameters obtained in step 4 are used to start a new iteration of the fitting procedure (from step 1 ).

The CPE parameters obtained from the fit are reported in Table 3.

A quantitative assessment of the CPE parameters can be appreciated in Fig. 1, where we report the CPE-computed charges of some configurations of the water and ethanol molecules, surrounded by a point charge, against the corresponding Mulliken charges.

As we can see, the CPE parameters provide excellent agreement for water (Fig. 1, panel A), while for ethanol (Fig. 1, panel B) larger deviations from quantum-chemical calculations are observed, especially for the methylene group. However, considering the simplicity of the united-atom model and the large interval in which the charges are scattered (e.g., the charge of the $\mathrm{O}$ in ethanol takes a value ranging from $-0.9 e$ to $-0.5 e$ ), the agreement with quantum-chemical calculations appears in general very satisfactory.

For the sake of completeness, the charge distributions for water and ethanol sites in bulk phase calculated along the MD runs, as well as the charge values calculated from the same MD runs with applying an external unitary electric field, are shown in Fig. 2.

It is important to remark that all MD simulations have been performed with a standard force field and hence the CPE charges account for the polarization only in a partial way being the dynamics produced by a non-polarizable force field. The charge distributions are featured

\section{Table 3}

Atomic parameters ( $\chi^{0}$, electronegativity, in $\mathrm{eV} / \mathrm{e} ; \eta^{0}$, hardness in $\mathrm{eV} / \mathrm{e}$ [2]) of the CPE sites for the water and ethanol molecules obtained from fitting the quantum-chemical data as described in the text

\begin{tabular}{lll}
\hline CPE site & $\chi^{0}$ & $\eta^{0}$ \\
\hline $\mathrm{H}$ & 2.00 & 23.96 \\
$\mathrm{O}$ & 10.00 & 22.89 \\
$\mathrm{LP}_{\mathrm{w}}$ & 7.87 & 25.14 \\
$\mathrm{CH}_{3}$ & 4.92 & 25.00 \\
$\mathrm{CH}_{2}$ & 1.57 & 25.00 \\
$\mathrm{O}$ & 10.56 & 13.40 \\
$\mathrm{H}$ & 6.33 & 14,20 \\
$\mathrm{LP}_{\mathrm{e}}$ & 11.23 & 15.37 \\
\hline
\end{tabular}

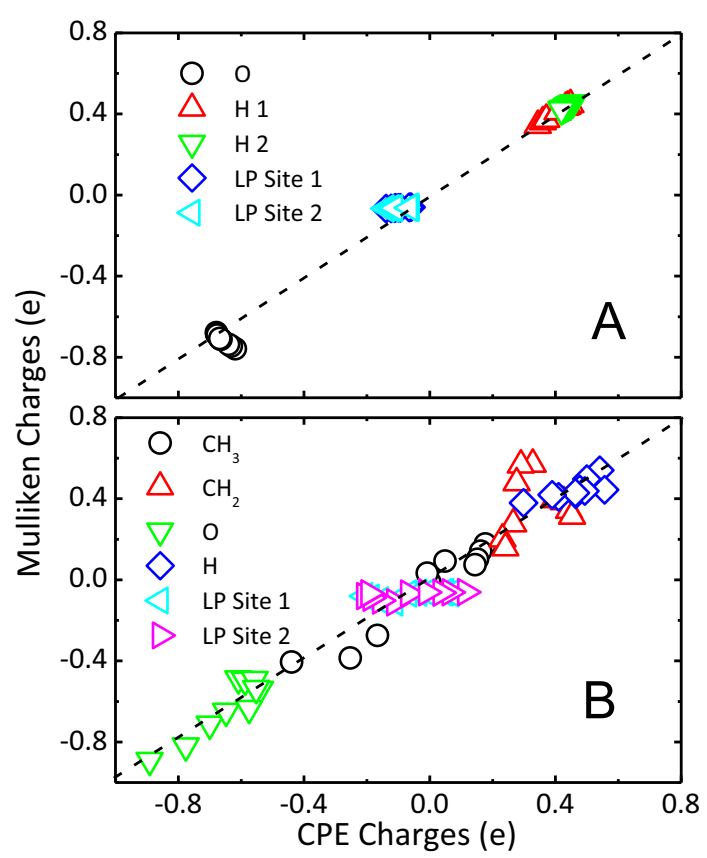

Fig. 1. Comparison between Mulliken and CPE charges for 10 water and 10 ethanol molecules (panels A and B, respectively) subject to an electric field produced by a point charge ( $q=1 \mathrm{e}$ ) randomly arranged around the molecule.

by a broadening as large as about $0.5 e$, especially for the $\mathrm{O}, \mathrm{H}$, and $\mathrm{LP}$ sites. The broadening is lower for alkyl groups, as expected from their globally smaller interactions with the environment with respect to the

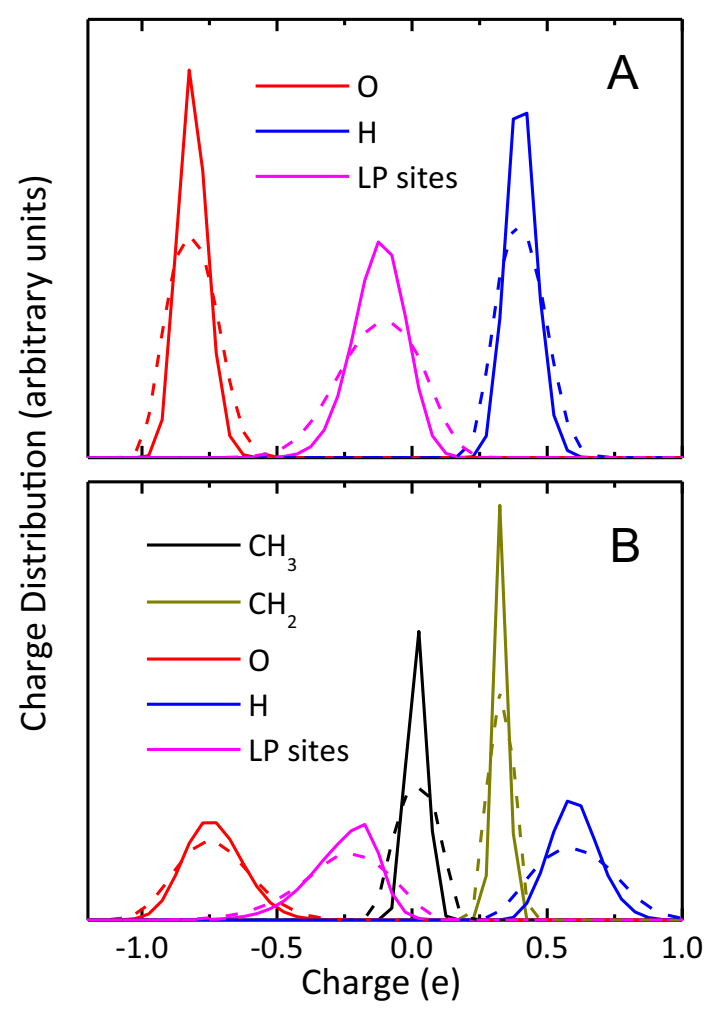

Fig. 2. Charge distributions computed from snapshots obtained by MD simulations of neat water (panel A) and neat ethanol (panel B). The continuous lines are related to the charges of the CPE sites (see legend) calculated without external electric field, while the dashed lines represent the corresponding charges calculated with a unitary external electric field. 
$\mathrm{O}$, hydroxy $\mathrm{H}$ and LP sites. The presence of an external electric field has the effect of increasing the broadening of the charge distributions of all molecular sites, keeping almost unchanged the average charge.

\section{Results and discussion}

\subsection{H-bonding}

Given that the usual radial distribution function provides average structural information, it is necessary to use alternative methods to achieve information on the local heterogeneities that could appear in the non-ideal mixtures [8]. When a molecule makes H-bonds in a neat liquid or in solution, it forms, together with its $\mathrm{H}$-bonded neighbors, a sort of $\mathrm{H}$-bond cluster, which usually shows a size distribution. A cluster can be isolated or to share one or more molecules with other clusters to give rise to a wider $\mathrm{H}$-bonded domain. In liquids formed by molecules that can form several $\mathrm{H}$-bonds either as $\mathrm{H}$ donor or as $\mathrm{H}$ acceptor, such a water or ethanol, the H-bond clusters are interconnected to give rise to an H-bond network extended through the whole bulk. Despite the complexity of the structure and dynamics of $\mathrm{H}$-bond clusters, structural classification is based on simple geometric criteria to determine if two molecules are H-bonded, neglecting any influence of the mean cluster lifetimes in the definition of those clusters. A quite common geometric criterion for $\mathrm{H}$-bonds of the type $\mathrm{O}-\mathrm{H} \cdots \mathrm{O}$, is based on distances and angles [68,69]. In particular, a H-bond is in place when the $0 \cdots \mathrm{O}$ distance is smaller than $3.5 \AA$, the $\mathrm{O} \cdots \mathrm{H}$ distance is smaller than $2.6 \AA$ and the angle defined by the $\mathrm{O} \cdots \mathrm{O}$ and $\mathrm{OH}$ directions is below the threshold of 30 degrees [70].

In Fig. 3, we quantify the extent of connectivity of the water and ethanol molecules for selected concentrations, in terms of water-water, water-ethanol, and ethanol-ethanol H-bonds.

In particular, for each type of $\mathrm{H}$-bond and for various concentrations, we report the fraction of molecules (in percentage) as a function of the number of neighboring H-bonded molecules. Note that, while for
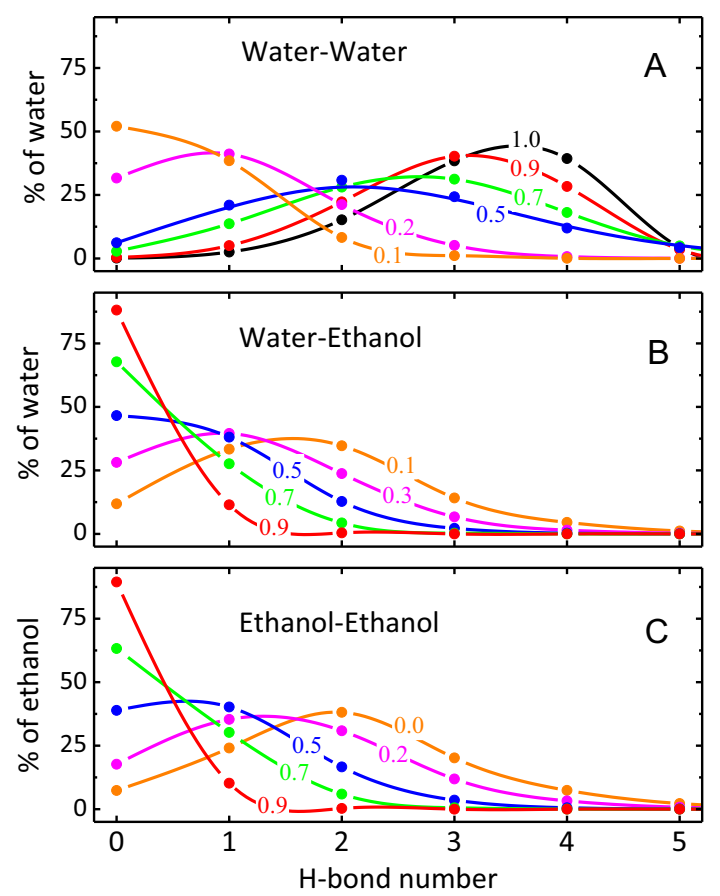

Fig. 3. Percentage of molecules of the type reported in the ordinate axis label as a function of the number of $\mathrm{H}$-bonds established as either $\mathrm{H}$ donor or $\mathrm{H}$ acceptor for selected values of $\chi_{w}$ (see the numbers on the colored lines). The statistics is reported for water-water (panel A), water-ethanol (panel B), and ethanol-ethanol (panel C) H-bond pairing. The lines are guides for the eyes. water-water and ethanol-ethanol H-bonds there is no ambiguity about the reference molecule to which the fraction refers to, in the case of the water-ethanol H-bonds, the fraction refers to water molecules. With increasing the ethanol concentration, there is an evident and expected reduction of the number of water molecules H-bonded to a reference water molecule, as it can be inferred from the left shift of the maximum of the distribution (Fig. 3, panel A). Below the water concentration of 0.5 , we observe a progressive increase in the number of water molecules that do not present any H-bond with any other water molecule. Considering the $\mathrm{H}$-bonds formed by an ethanol molecule with other ethanol molecules in neat liquid ethanol (Fig. 3, panel C), we note a clear maximum at two H-bonds per ethanol molecule, in agreement with the calculations by Saiz et al. [71]. In waterrich mixtures, the number of $\mathrm{H}$-bonds formed by a water molecule with ethanol molecules is low (Fig. 3, panel B), because most of the water molecules are involved in water-water H-bonds. The fraction clearly increases with increasing the ethanol content. In any case, even in ethanol-rich mixtures, a significant amount of water molecules (about $40 \%$ with respect to the total amount of water) remains H-bonded to at least one other water molecule (Fig. 3, panel A). Moreover, from the ethanol-ethanol H-bond histograms (Fig. 3, panel C), it is possible to see how fast the ethanol H-bond network profile changes in mixtures with relatively small amounts of water added in. The fast change in the pure-like behavior of the liquid ethanol with a small amount of water was observed experimentally in the pre-edge region of alcohol X-ray absorption spectra [7]. For instance, at $\chi_{w}=0.2$, the distribution of $\mathrm{H}$-bonded ethanol-ethanol cluster size becomes broader, and with a further increase of water content in the mixture there is an increase in the number of ethanol molecules that do not make $\mathrm{H}$ bonds with any other ethanol molecule. A very similar behavior was also found in MD simulations of water-methanol mixtures [72].

A picture of $\mathrm{H}$-bond clustering, complementary to that reported in Fig. 3, is provided in Fig. 4, where we collect the data of Fig. 3 in terms
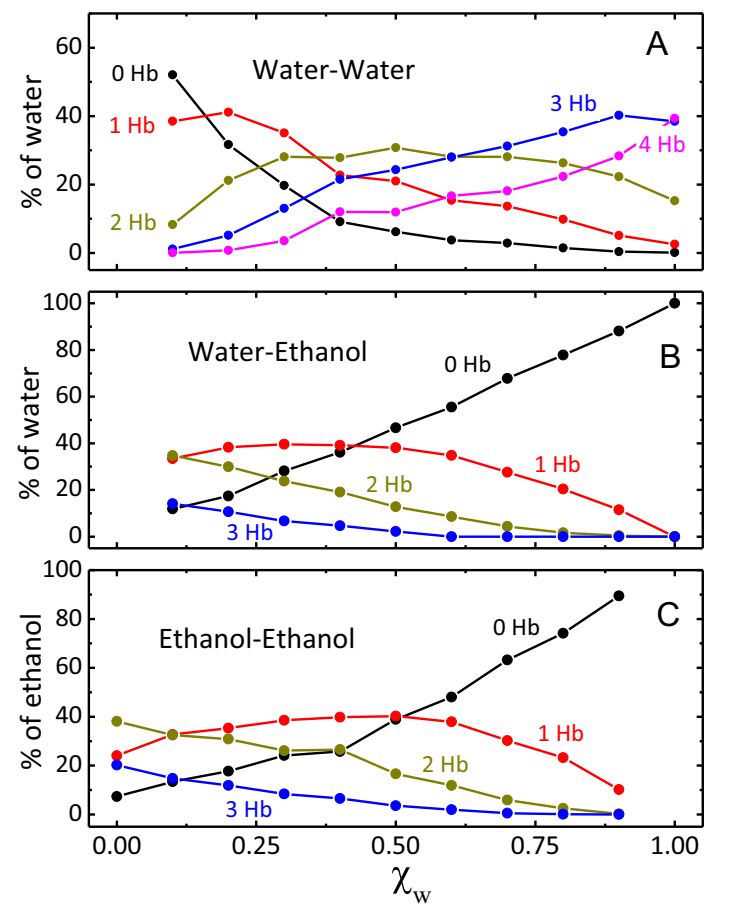

Fig. 4. Percentage of molecules of the type reported in the ordinate axis label forming an established number of $\mathrm{H}$-bonds (see the labeling of the colored lines) as a function of the water molar fraction. The statistics is reported for water-water (panel A), waterethanol (panel B) and ethanol-ethanol (panel C) H-bond pairing. The lines are guides for the eyes. 
of concentration instead of the number of H-bonds. In Fig. 4, panel A, we show the behavior of water H-bonded clusters.

Decreasing the water content from the pure water sample, there is an almost constant depletion of large-size clusters (molecules forming 3 and 4H-bonds) and an increase in the smaller ones, until $\chi_{w} \sim 0.4$. With further increase of ethanol in the mixture, we note: (i) a quite evident increase of the depletion rate of large H-bond clusters, (ii) an important increase and persistence of $\mathrm{H}$-bonded water dimers (the number of water molecules forming one $\mathrm{H}$-bond with another water molecule) and (iii) a clear increment in the formation rate of non-Hbonded water molecules. In Fig. 4, panel B, we report the percentage of $\mathrm{H}$-bond clusters formed by a single water molecule $\mathrm{H}$-bonded to 0 up to 3 ethanol molecules along the concentration. Departing from pure water, we note three relevant aspects: $(i)$ an almost linear decrease of the percentage of water molecules non-H-bonded with ethanol molecules along the concentration range, (ii) an increase of water-ethanol H-bonded dimers ( 1 water molecule H-bonded to only 1 ethanol molecule) until $\chi_{w} \sim 0.4$, where a flat behavior occurs and (iii) a continuous increase of the amount of the H-bond clusters formed by a single water molecule and 2 and 3 ethanol molecules. In panel C, we report the percentage of $\mathrm{H}$-bonded clusters of ethanol alone along with the concentration of the mixtures. Departing from pure ethanol, a reduction of largest ethanol H-bonded clusters and a concomitant increase of smaller ethanol H-bonded clusters take place until $\chi_{w} \sim 0.4$, where a further increment of water leads to an increase of the formation rate of isolated ethanol molecules and of the depletion rate of H-bond clusters formed by 2 ethanol-ethanol H-bonds, as well. Hydrophobic interactions act in order to sustain water clusters even in ethanol rich mixtures and, at the other side, a great number of hydrated single ethanol molecules in water-rich mixtures. These clear opposite trends appear to cross at $\chi_{w}$ $\sim$ 0.4. According to the observed behavior of the hydroxy proton of EtOH [1]H NMR chemical shift resonance [73], there are clearly major changes in the collective structure-dynamical properties of ethanolwater mixtures ascribed to the changes on the H-bonds strength. MD simulations also suggest a quite similar behavior of the dynamics of the $\mathrm{H}$-bond along the concentration in methanolic aqueous mixtures [74,75].

\subsection{Time correlation functions of the polarizability anisotropy}

Information about the dynamical behavior of the collective polarizability anisotropy for liquid water calculated using the CPE model can be gained from Fig. 5, where we report the polarizability anisotropy TCFs, $\Psi(t)$, as well as its components $\Psi^{M M}(t), \Psi^{M I}(t)$ and $\Psi^{I I}(t)$, for neat liquid water.
For the sake of comparison with a model less refined than CPE, the TCFs have also been computed using the dipole-induced-dipole (DID) model [76], exploiting the same MD trajectories. The DID model allows to compute directly the interaction-induced polarizability, $\Pi^{I}$. The $\Pi^{M}$ component is simply evaluated by summing all the polarizability tensors of the molecules in the sample. A limitation of the DID model is that, since the polarizability is treated as concentrated in the center of mass of the molecule, it neglects the details of the shape of the molecular charge distribution and hence may fail in representing accurately the interactions between molecules. This aspect is particularly important at short distances, where the molecular shape plays a fundamental role. In spite of this, here we want to explore how a detailed description of the molecular shape, included implicitly in the CPE model, may affect the qualitative behavior of the polarizability time relaxation. The results obtained from the DID model are reported in Fig. 5, panel B. There is an overall agreement between the current calculated functions, using the explicit form for the interaction-induced part, with previous data reported in the literature for pure liquid water [17]. According to Fig. 5, the polarizability relaxation of liquid water $\Psi(t)$ is characterized by a fast decay at short time, followed by a slightly damped oscillatory behavior that converges into a simple exponential decay [77] at the rotation-diffusion limit. As we can observe in the figure, the dumped oscillatory time-dependence is mainly originated by the permanent contribution $\Psi^{M M}(t)$. This behavior is expected because this function is sensitive to the short-range force pulses experimented by the molecules mainly from the first neighbor shell of $\mathrm{H}$-bonded molecules. Since the CPE parameters are able to reproduce the molecular gasphase polarizability, the function $\Psi^{M M}(t)$, which mainly depends on the orientational dynamics of the molecules, does not differ significantly from that computed using the DID model. The DID model predicts an important degree of participation of $\Psi^{I I}(t)$ to the overall relaxation of the anisotropic polarizability, approximately half of the observed contribution due to the permanent part. In the case of the CPE model, the contribution from the cross part, $\Psi^{M I}(t)$, is slightly greater with respect to DID, even if the relaxation-time profiles are very similar to those observed for the DID model. The interaction-induced part is mainly determined by the long-range interactions, so we observe a more smooth and exponential decay and a small and positive contribution of the cross part, very similar to the findings of Bursulaya et al. [17]. In conclusion, despite the important differences between the DID and CPE approaches, our results suggest that even a simple model as the DID one, is able to capture the basic qualitative features of the polarizability anisotropy relaxation.

In Fig. 6, panel A, we report the polarizability anisotropy TCFs, $\Psi(t)$, normalized to the unity, for mixtures with selected concentrations.
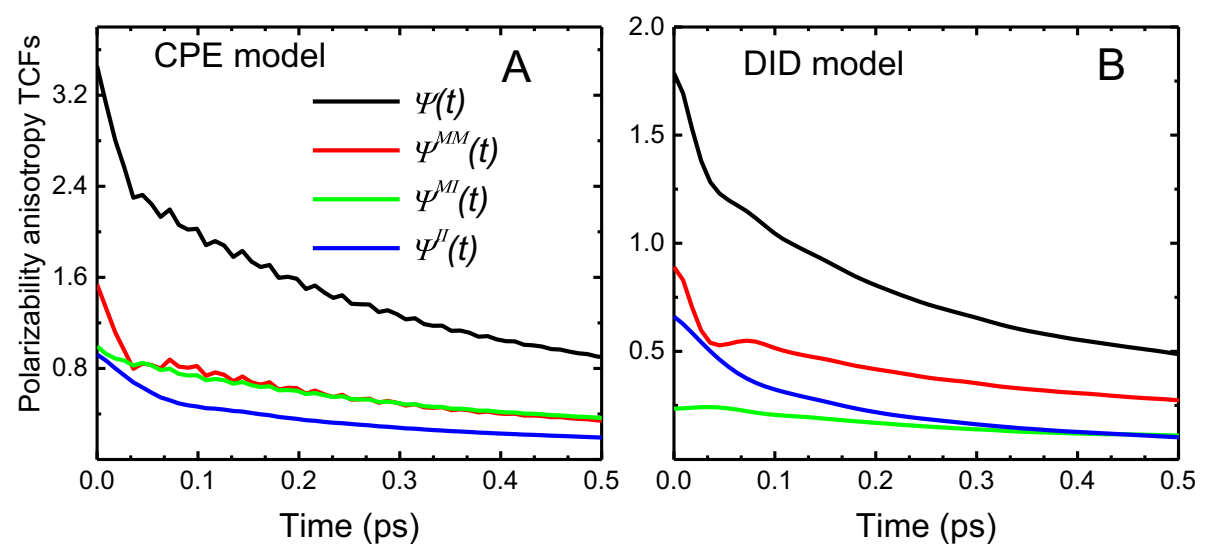

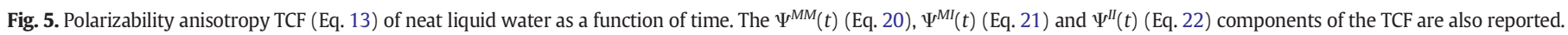
Calculations using the CPE and the DID models are reported in the panels A and B, respectively. 

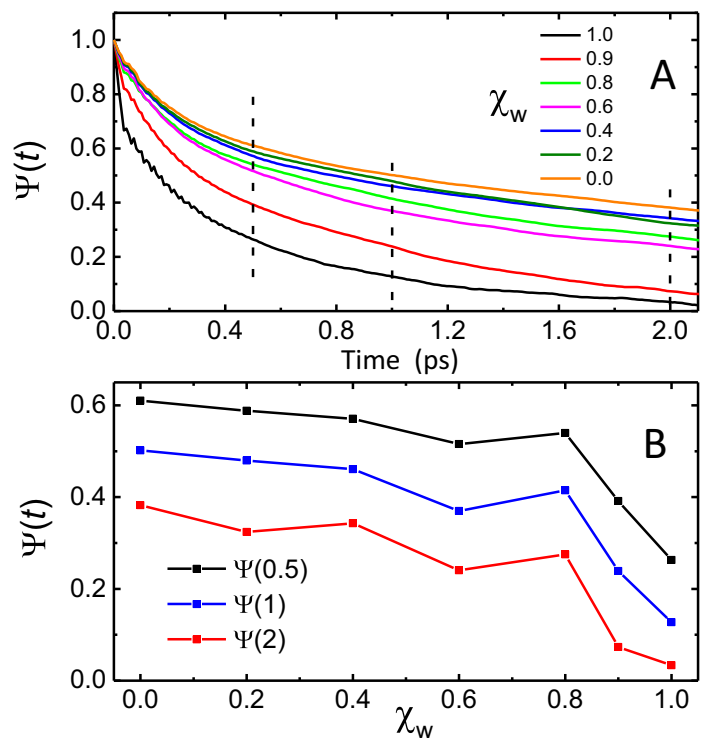

Fig. 6. Panel A: polarizability anisotropy TCF (Eq. 13) normalized to the unity as a function of time computed for various concentrations of the mixture (see legend). Panel B: polarizability anisotropy TCF for three selected times $(0,5,1$ and $2 \mathrm{ps}$, see legend) as a function of the mixture concentration. The lines are guides for the eyes. Calculations have been performed using the CPE model.

With increasing the amount of ethanol, the anisotropy relaxation of the system becomes progressively slower, as also found in previous experimental studies reporting the composition dependence of collective relaxation properties in ethanol-water mixtures, like the dipole moments along the dielectric relaxation [78], NMR proton relaxation [79], as well as theoretical works using MD simulations [80]. As a straight consequence of the fact that the molecular polarizability of ethanol is almost five times greater than the polarizability of water, the behavior of $\Psi(t)$ is dominated by the contribution of ethanol, appearing influenced by the water dynamics only in water-rich mixtures (see discussion below). In fact, the general trend of $\Psi(t)$ as a function of the concentration is clearly non-monotonic, being more sensitive to changes of concentration in water-rich mixtures. This can be observed more clearly in Fig. 6, panel $\mathrm{B}$, where we report the values of $\Psi\left(t^{\prime}\right)$ for three representative times, $t$ $'=0.5,1,2 \mathrm{ps}$, as a function of the mixture concentration. We note that, in a small range of $\chi_{w}$, specifically from 0.8 to 1.0 , the change of $\Psi\left(t^{\prime}\right)$ is about 0.3 , while in the wide range of concentration $0.0-0.8$, the function $\Psi\left(t^{\prime}\right)$ varies by only about 0.1 . As we can note from the curves related to $\Psi(0.5), \Psi(1)$ and $\Psi(2)$, such a behavior occurs without appreciable differences through the whole-time interval. The trend of $\Psi\left(t^{\prime}\right)$ above $\chi_{w} \simeq 0.8$ (Fig. 6, panel B) shows a relevant dependence on the concentration, whereas it becomes nearly constant below the concentration of 0.8 . The interpretation of such a behavior is not obvious. From one side, as also remarked above, we may note that the polarizability of ethanol is about five times greater than that of water. This may imply that polarization anisotropy decay is mainly due to ethanol already in water-rich mixtures. At very low concentrations of ethanol, any addition of ethanol makes slower the system dynamics according to the intrinsically low dynamics of ethanol itself. At a concentration of $\chi_{w} \simeq 0.8$, the contribution of ethanol to the dynamics may become dominant and a further decrease of $\chi_{w}$ gives rise only to a modest change of the system dynamics. From the other side, a more mechanistic interpretation can be invoked to explain the fast change of $\Psi\left(t^{\prime}\right)$ above $\chi_{w} \simeq 0.8$. Indeed, the change could be due to an intrinsic slowing down of the water dynamics arising from a reinforcement of $\mathrm{H}$-bonding in solution. In fact, in water-rich mixtures, $\chi_{w} \sim 0.85$, there are both experimental $[81,82]$ and theoretical evidences $[2,83]$ for a counterintuitive trend of the water molecules to keep or even to reinforce their networking

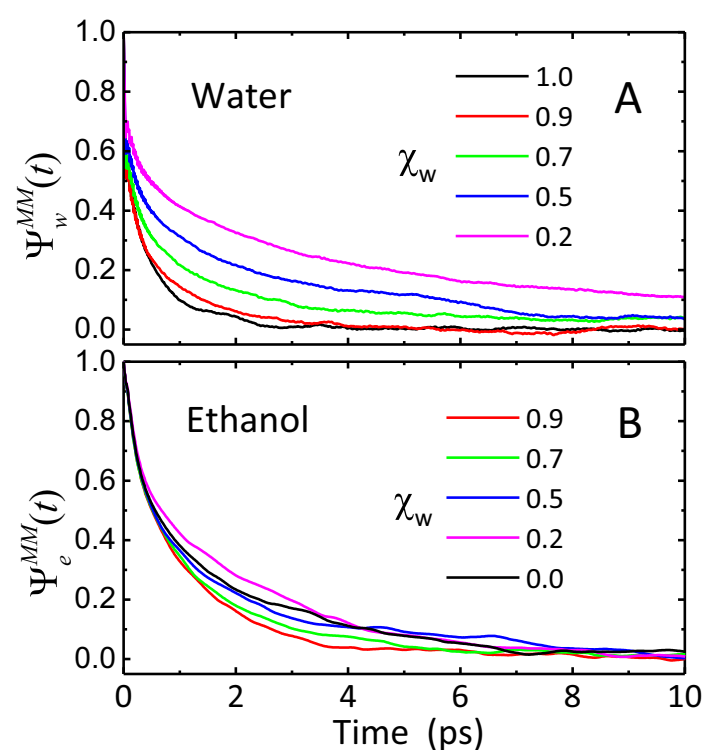

Fig. 7. Permanent component $\Psi^{M M}(t)$ (Eq. 20; normalized to the unity) of the polarizability anisotropy TCF, $\Psi(t)$, as a function of time computed for various concentrations of the mixture (see the legend). The calculations have been performed considering only water molecules (panel A) and only ethanol molecules (panel B). Calculations have been performed using the CPE model.

interactions with respect to the neat liquid. This interpretation arises from observing a maximum in the sound velocity, which suggests a greater degree of connectivity between the water molecules and, as a consequence, the segregation of the ethanol molecules.

Typically, in molecular systems at ordinary temperature and pressure, the permanent/orientational contribution is the main responsible for the polarizability anisotropy relaxation. The TCFs considering exclusively the permanent contribution from water, $\Psi_{w}^{M M}(t)$, and ethanol molecules, $\Psi_{e}^{M M}(t)$,along selected concentrations are displayed in Fig. 7.

These functions indicate that the orientational dynamics of the water molecules is clearly much more sensitive to the variations of concentration than the corresponding orientational dynamics of the ethanol molecules. In fact, the latter appears to be moderately affected by a change of concentration (Fig. 7, panel B).

The results of Figs. 6 and 7 suggest that the slowing down of the dynamics observed going from water-rich to ethanol-rich solutions is due basically to the increase of ethanol content rather than to a change of dynamics of the ethanol itself. In water-rich solutions (above $\chi_{w} \simeq$ 0.8 ), the dynamics is fast because it is dominated by water. When ethanol is added to the solution, owing to its higher molecular polarizability with respect to water, the system dynamics becomes more and more slow, approaching that of pure ethanol. In this dynamics vs. concentration evolution, we envisage the onset of a dynamical transition occurring at relatively low concentrations of ethanol, namely $\chi_{w} \sim 0.8$ (see Fig. 6, panel B). Due to the differences in the inertial moments, the ethanol reorientation dynamics is almost insensitive to concentration in comparison with that of water molecules, whose dynamics is significantly affected by the presence of ethanol.

In Fig. 8, we report the TCFs of the interaction-induced contribution to the anisotropic polarizability relaxation, $\Psi^{I I}(t)$, for some selected mixture concentrations.

As observed for $\Psi(t)$ (Fig. 6), also this function shows a clear increase of the relaxation times departing from pure water with increasing amounts of ethanol. This increase is not monotonic and we observe that larger jumps into the relaxation times take place in water-rich solutions, with an evident trend of reducing the jump size with increasing the ethanol concentration. It is reasonable to ascribe such a behavior to the same mechanism discussed above for $\Psi(t)$. 


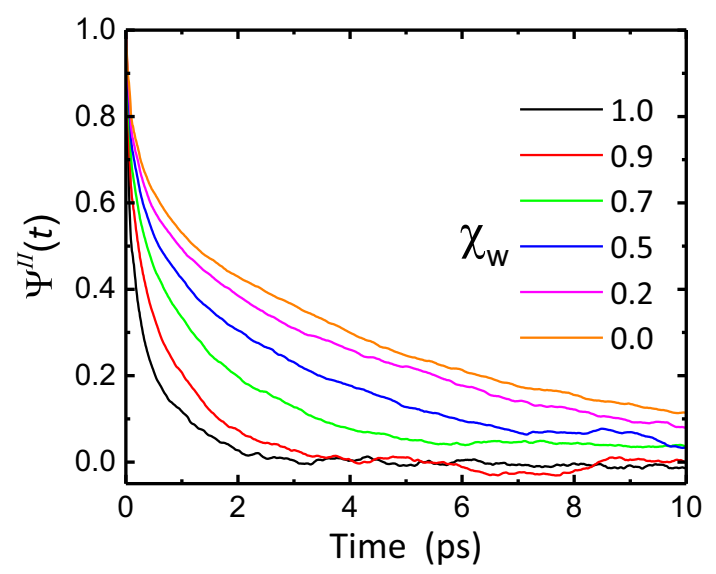

Fig. 8. Interaction-induced component $\Psi^{I I}(t)$ (Eq. 22; normalized to the unity) of the polarizability anisotropy TCF $\Psi(t)$, as a function of time computed for various concentrations of the mixture (see the legend). Calculations have been performed using the CPE model.

\subsection{Anisotropic Raman scattering}

The vibrational components seen in the low-frequency anisotropic Raman spectra are usually reported on the susceptibility form, $\chi^{\prime \prime}(\omega)$, obtained here using the equation $[84,85]$

$\chi^{\prime \prime}(\omega)=\frac{I(\omega)}{[\exp (h \omega / k T)-1]^{-1}+1}$

where $I(\omega)$ is the total Raman intensity (Fourier transform of Eq. $13, \Psi_{x y}$ $(t)$ ). According to the available low-frequency Raman data [86,87], far IR [88] absorption spectra, as well as theoretical works [86,87,89-91], the reduced low-frequency Raman spectra of pure liquid water at room temperature is composed mainly by two broad and strongly overlapped components centered at about 60 and $170 \mathrm{~cm}^{-1}$ for pure water and 60 $\mathrm{cm}^{-1}$ and $70 \mathrm{~cm}^{-1}$ for pure ethanol, respectively assigned to restricted translations perpendicular and along the $\mathrm{O}-\mathrm{H} \cdots \mathrm{O}$ direction or, in other

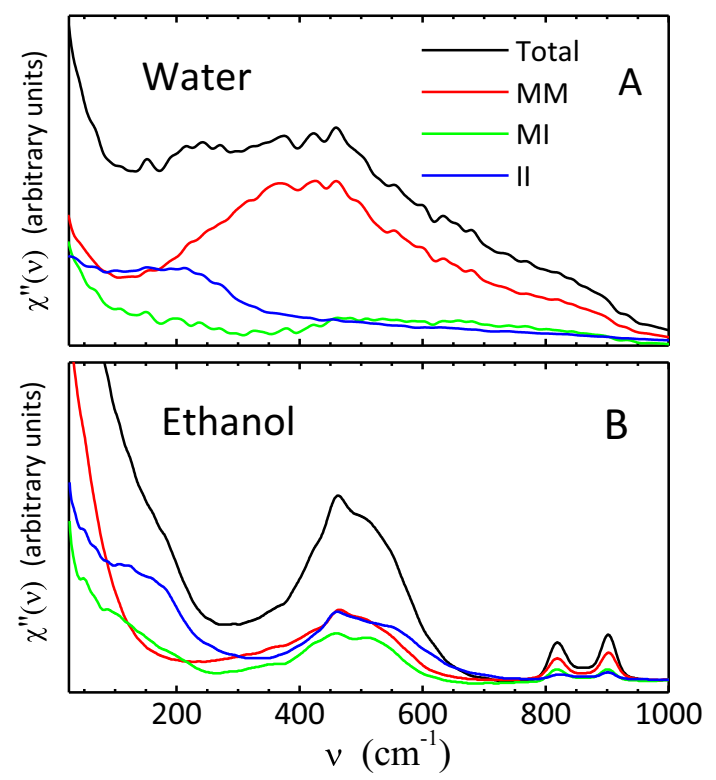

Fig. 9. Low-frequency behavior of $\chi^{\prime \prime}(\omega)$ and its MM, MI and II components (see the legend) for water and ethanol neat liquids (panels A and B, respectively), calculated as described in Section 2.B. Calculations have been performed using the CPE model. words, to collective motions consisting of bending and stretching of the H-bonds.

The $\chi^{\prime \prime}(\omega)$ intensity and the corresponding contributions from the permanent (MM), cross (MI), and interaction-induced (II) mechanisms for pure ethanol and pure water are shown in Fig. 9.

For the pure liquid water (Fig. 9, panel A), the total spectral shape is influenced by the II component in the very low frequency region (up to $250 \mathrm{~cm}^{-1}$ ), where we note at least two overlapped components, one centered at about $220 \mathrm{~cm}^{-1}$ and the second falling below $100 \mathrm{~cm}^{-1}$. Based on the agreement between the experimental low-frequency Raman spectra and the simulated spectral density, it is commonly accepted $[92,93]$ that, at low frequencies, the experimental data are affected significantly by the II mechanism, which is consistent with our observations. The MM component is clearly the most intense and broad contribution to the spectral density falling above $\sim 250 \mathrm{~cm}^{-1}$. This component usually matches with experimental bands assigned as hindered orientational motions (librations) about the molecular axes, since those bands are strongly shifted with deuteration [94]. In the case of pure ethanol (Fig. 9, panel B), the II mechanism appears much more important with respect to water, especially in the frequency range $100-250 \mathrm{~cm}^{-1}$, where it provides the greatest contribution to the spectral density. In addition, its contribution to the band around $500 \mathrm{~cm}^{-1}$ is also relevant, whereas in water it enters practically as a spectral background. Moreover, it is quite surprising that the MM component in the ethanol spectral density does not dominate the other components like in water, apart from at very low frequencies. In fact, above $250 \mathrm{~cm}^{-1}$, the spectral density appears to receive nearly equal contributions from MM, MI and II mechanisms and is characterized by the presence of two bands related to the ethanol intramolecular bending motions [95].

In Fig. 10, we report the MM component of the reduced spectral density for some relevant concentrations, by limiting the polarizability calculation to the water molecules alone.

As discussed above, the permanent anisotropy relaxation results into the most broad and widespread contribution to the low frequency spectrum of pure water. This contribution however appears not to be the only determinant in the experimental Raman data at very low frequencies, where induction mechanisms become significant. Concerning librational motions falling above $200 \mathrm{~cm}^{-1}$, we note the occurrence of strongly overlapped bands for all considered concentrations, like in pure water (Fig. 9, panel A), which form a very broad envelope, ranging through a frequency interval of about $800 \mathrm{~cm}^{-1}$. The strong overlap between such bands precludes a careful analysis. In spite of this, as only the permanent polarizability component of water molecules is accounted for in the calculation, we may ascribe the observed

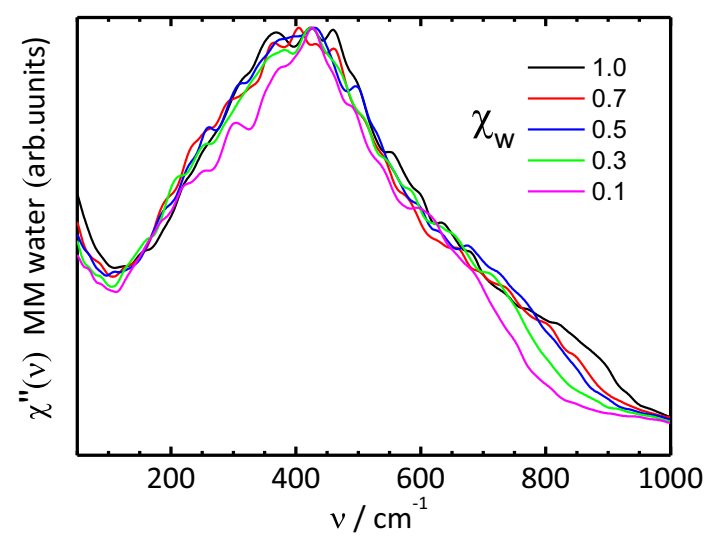

Fig. 10. Low-frequency behavior of the MM component of $\chi^{\prime \prime}(\omega)$ computed considering only water molecules. The results for selected concentrations are reported (see the legend). Calculations have been performed using the CPE model. 


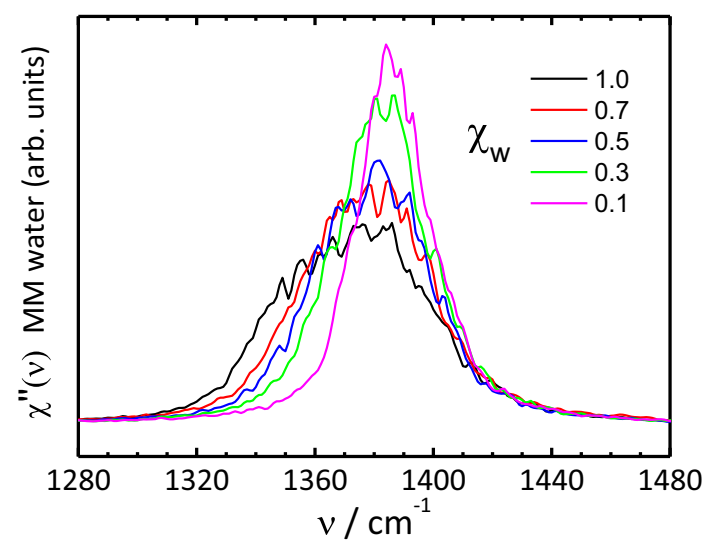

Fig. 11. High-frequency behavior (bending-mode region) of the MM component of $\chi^{\prime \prime}(\omega)$ computed considering only water molecules. The results for selected concentrations are reported (see the legend). Calculations have been performed using the CPE model.

broadening mainly to the dynamics of those molecules, basically associated to the fact that the water molecules undergo more or less hindered rotational motions in several different configurational patterns (inhomogeneous broadening). With increasing the ethanol concentration, we can observe a small reduction of the width of the band envelope, especially at frequencies above $750 \mathrm{~cm}^{-1}$, suggesting for a slightly more homogeneous environment in ethanol-rich mixtures, namely a more localized distribution of the librational frequencies.

While the environment of the water molecules does not affect much the spectral inhomogeneity at low frequencies in solutions with different concentration (see Fig. 10 and related discussion), a greater effect of the environment is instead observed for the $v_{2}$ water bending mode. This can be realized observing the MM component of the reduced spectral density reported in Fig. 11 for solutions at various concentrations (also in this case, as for the data of Fig. 10, the calculation is limited to water molecules alone).

The evolution of the bandwidth of the water bending mode through the whole concentration range suggests that, by moving from pure water to pure ethanol, spectral homogeneity enhances. This effect can arise from the lowering of the interactions established by water with its environment when the amount of ethanol increases. Such a feature is consistent with the fact that H-bonding strength is smaller for water-ethanol pairs than for water-water pairs and that bending mode depends significantly on the H-bond strength. It is worthwhile

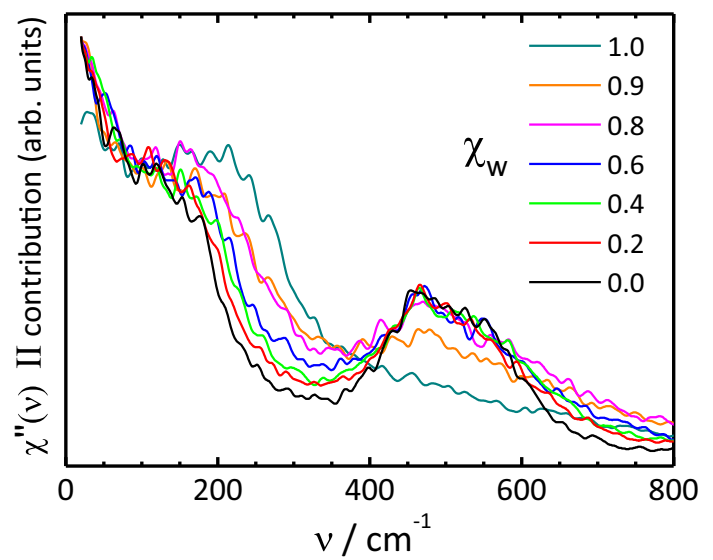

Fig. 12. Low-frequency behavior of the II component of $\chi^{\prime \prime}(\omega)$ for selected concentrations (see the legend). Calculations have been performed using the CPE model. to note that, due to the greater value of the gas-phase polarizability of the ethanol molecule with respect to water, this information could be not easy to see in the experimental spectra.

In Fig. 12, we report the reduced II spectral density, normalized to the unity, for several concentrations.

As expected, the typical low-frequency main bands are broad and strongly overlapped. Moreover, the number of the main bands, their position and the behavior of the spectra match qualitatively the available experimental data $[96,97]$. The most clear changes are on the relative intensity of the bands due to the variation of the relative number of molecules along the concentration range. In particular, in agreement with experiments [96,97], in the calculated reduced II spectral density we observe a relevant enhancement of the intensity around $250 \mathrm{~cm}^{-1}$ with increasing the water content in the mixture. Moreover, we also notice a relevant dependence of the intensity of the band at about $500 \mathrm{~cm}^{-1}$ on the concentration of the mixture. Consistently with the experimental trend, such a band is strong in ethanol rich mixtures, while gradually disappearing when ethanol concentration lowers. As previously stated, the II polarizability relaxation appears to be the main mechanism that determines the shape of the bands in the low-frequency range, except for the presence of a persistent relaxational component below $80 \mathrm{~cm}^{-1}$, more clearly seen in the mixtures and in the pure ethanol (see Fig. 9, panel B). Due to the difference between the gas-phase polarizabilities of water and ethanol, even in water-rich mixtures, the spectral density is strongly influenced by the ethanol contribution. In fact, as noted above, by increasing the ethanol concentration, we observe a fast and progressive depletion of the contribution of water to the spectral density around $250 \mathrm{~cm}^{-1}$ and the emergence of the typical librational bands assigned to ethanol from 350 to $650 \mathrm{~cm}^{-1}$. A possible explanation is based on considering the total reduced spectral density as a linear combination of the spectral densities of the pure systems and the differences between the real spectrum and the one resulting from the spectral combination of the pure systems as due to the so-called cooperative dynamics (for water and tert-butyl alcohol see Ref. 98, for waterethylene glycol see Ref. 99). Despite the qualitative agreement, given the aforementioned features of the low-frequency contributions, we acknowledge that the typical differences of the real spectrum and the linear combination of the pure water and ethanol contributions are very subtle even for up-to-date experimental techniques [100].

\section{Concluding remarks}

MD simulations were performed to investigate structural and dynamical properties of water-ethanol mixtures along the entire concentration range from neat water to neat ethanol. We make use of appropriate occurrence histograms to unveil structural heterogeneity related to H-bond network dynamics. Using the geometrical definition of $\mathrm{H}$-bond, it was possible to quantify carefully the H-bonded water clusters depletion rate, and the formation of water-ethanol as well as the ethanol clusters with increasing amounts of ethanol. We can remark the following. (i) The strength and persistence of the water-water $\mathrm{H}$-bonded network in comparison to the weakness of the ethanolethanol counterpart, (ii) a peculiar threshold composition $\left(\chi_{w} \approx 0.4\right)$ from where the water molecules experience an increasing less perturbative and a greater environmental homogeneity due to the effective dilution of the water molecules in ethanol rich mixtures. It is worth to mention that the threshold concentration, could be experimentally achieved, since the theoretical Raman bandshapes as well as the position of water intramolecular bands appears to be quite sensitive to such environmental changes.

For the polarizability relaxation analysis, two sets of $\mathrm{CPE}$ parameters for both 5-sites water and a 6-sites coarse-grained ethanol molecules have been obtained by fitting the electronic properties calculated using ab initio methods. The polarizability TCFs computed using the CPE model agree qualitatively with the results obtained with a dipole- 
induced-dipole model, except for the distinct degree of reorientational mechanism into the relaxation of the total polarizability, since the CPE model appears to be more sensitive to the collision mechanisms. The polarizability TCFs from the ethanol molecules are characterized by a slow and nearly constant decay rate along all the concentration range. In contrast, the corresponding TCFs calculated for the water molecules shows a wide range of decay rate, meaning a great spectroscopic sensibility to the environmental changes according to the composition. Controversially, since the gas-phase polarizability of the ethanol molecule is about five times greater than the corresponding value of the water molecule, the ethanol contribution dominates the low-frequency spectral even in water-rich mixtures. Finally, the calculated interactioninduced number of the main bands as well as their intensities agrees qualitatively with the available low-frequency reduced Raman spectra.

\section{Declaration of Competing Interest}

The authors declare that they have no known competing financialinterestsor personal relationships that could have appeared to influence the work reported in this paper.

\section{Acknowledgements}

The present study is funded by the MIUR-Italy (Progetto Dipartimenti di Eccellenza 2018 - 2022 allocated to the Department of Chemistry - Ugo Schiff - of the University of Firenze, Italy).

\section{References}

[1] M.D. Elola, B.M. Ladanyi, J. Chem. Phys. 125 (2006) 184506.

[2] M. Mijaković, B. Kežić, L. Zoranić, F. Sokolić, A. Asenbaum, C. Pruner, E. Wilhelm, A. Perera, J. Mol. Liq. 164 (2011) 66.

[3] E.J.W. Wensink, A.C. Hoffmann, P.J. Van Maaren, D. Van Der Spoel, J. Chem. Phys. 119 (2003) 7308

[4] M. Matsugami, R. Yamamoto, T. Kumai, M. Tanaka, T. Umecky, T. Takamuku, J. Mol. Liq. 217 (2016) 3.

[5] O. Gereben, L. Pusztai, J. Phys. Chem. B 119 (2015) 3070

[6] J.A. Larkin, J. Chem. Thermodyn. 7 (1975) 137.

[7] R.K. Lam, J.W. Smith, R.J. Saykally, J. Chem. Phys. 144 (2016) 191103.

[8] K. Takaizumi, T. Wakabayashi, J. Solut. Chem. 26 (1997) 927.

[9] T.A. Dolenko, S.A. Burikov, S.A. Dolenko, A.O. Efitorov, I.V. Plastinin, V.I. Yuzhakov, S.V. Patsaeva, J. Phys. Chem. A 119 (2015) 10806.

[10] S. Alavi, S. Takeya, R. Ohmura, T.K. Woo, J.A. Ripmeester, J. Chem. Phys. 133 (2010), 074505,

[11] Y.M. Zelenin, Zh. Strukt, Khimii 44 (2003) 155.

[12] R. Chelli, S. Ciabatti, G. Cardini, R. Righini, P. Procacci, J. Chem. Phys. 111 (1999) 4218.

[13] R. Chelli, S. Ciabatti, G. Cardini, R. Righini, P. Procacci, J. Chem. Phys. 112 (2000) 5515.

[14] R. Chelli, G. Cardini, P. Procacci, R. Righini, S. Califano, A. Albrecht, J. Chem. Phys. 113 (2000) 6851

[15] R. Chelli, G. Cardini, M. Ricci, E.P. Bartolini, R. Righini, S. Califano, Phys. Chem. Chem. Phys. 3 (2001) 2803.

[16] M. Paolantoni, B.M. Ladanyi, J. Chem. Phys. 117 (2002) 3856.

[17] B.D. Bursulaya, H.J. Kim, J. Chem. Phys. 109 (1998) 4911

[18] B.T. Thole, Chem. Phys. 59 (1981) 341.

[19] P.A. Madden, M. Wilson, F. Hutchinson, J. Chem. Phys. 120 (2004) 6609.

[20] W.J. Glover, P.A. Madden, J. Chem. Phys. 121 (2004)

[21] O. Wan, L. Spanu, G.A. Galli, F. Gygi, J. Chem. Theory Comput. 9 (2013) 4124.

[22] M. Pagliai, C. Cavazzoni, G. Cardini, G. Erbacci, M. Parrinello, V. Schettino, J. Chem. Phys. 128 (2008) 224514.

[23] A. Wallqvist, B.J. Berne, J. Phys. Chem. 97 (1993) 13841.

[24] P. Bultinck, W. Langenaeker, P. Lahorte, F. De Proft, P. Geerlings, C. Van Alsenoy, J.P. Tollenaere, J. Phys. Chem. A 106 (2002) 7895.

[25] J.L. Banks, G.A. Kaminski, R. Zhou, D.T. Mainz, B.J. Berne, R.A. Friesner, J. Chem. Phys. 110 (1999) 741.

[26] R. Chelli, P. Procacci, R. Righini, S. Califano, J. Chem. Phys. 111 (1999) 8569.

[27] P. Itskowitz, M.L. Berkowitz, J. Phys. Chem. A 101 (1997) 5687.

[28] W.J. Mortier, S.K. Ghosh, S. Shankar, J. Am. Chem. Soc. 108 (1986) 4315.

[29] R.F. Nalewajski, Int. J. Quantum Chem. 42 (1992) 243.

[30] A.K. Rappe, W.A. Goddard, J. Phys. Chem. 95 (1991) 3358.

[31] D.M. York, W. Yang, J. Chem. Phys. 104 (1996) 159.

[32] R. Chelli, P. Procacci, J. Chem. Phys. 117 (2002) 9175.

[33] R. Chelli, M. Pagliai, P. Procacci, G. Cardini, V. Schettino, J. Chem. Phys. 122 (2005), 074504,

[34] R.G. Parr, R.G. Pearson, J. Am. Chem. Soc. 105 (1983) 7512.
[35] R.G. Parr, W. Yang, Density-Functional Theory of Atoms and Molecules, Oxford University Press, New York, 1989.

[36] R.F. Nalewajski, J. Phys. Chem. 89 (1985) 2831.

[37] J. Korchowiec, R.F. Nalewajski, Int. J. Quantum Chem. 44 (1992) 1027.

[38] Z.-Z. Yang, C.-S. Wang, J. Phys. Chem. A 101 (1997) 6315.

[39] K.T. No, O.Y. Kwon, S.Y. Kim, K.H. Cho, C.N. Yoon, Y.K. Kang, K.D. Gibson, M.S. Jhon, H.A. Scheraga, J. Phys. Chem. 99 (1995) 13019.

[40] R. Heidler, G.O.A. Janssens, W.J. Mortier, R.A. Schoonheydt, J. Phys. Chem. 100 (1996) 19728

[41] R. Winkler, S.T. Pantelides, J. Chem. Phys. 106 (1997) 7714.

[42] C.A. Reynolds, J.W. Essex, W.G. Richards, J. Am. Chem. Soc. 114 (1992) 9075.

[43] J. Cioslowski, M. Martinov, J. Phys. Chem. 100 (1996) 6156.

[44] B.J. Berne, R. Pecora, Dynamic Light Scattering, Wiley, New York, 1976.

[45] L.C. Geiger, B.M. Ladanyi, Chem. Phys. Lett. 159 (1989) 413.

[46] J.S. Friedman, M.C. Lee, C.Y. She, Chem. Phys. Lett. 186 (1991) 161

[47] J.S. Friedman, C.Y. She, J. Chem. Phys. 99 (1993) 4960.

[48] H.J.C. Berendsen, J.R. Grigera, T.P. Straatsma, J. Phys. Chem. 91 (1987) 6269.

[49] V. Hornak, R. Abel, A. Okur, B. Strockbine, A. Roitberg, C. Simmerling, Proteins Struct. Funct. Bioinforma. 65 (2006) 712.

[50] C.I. Bayly, P. Cieplak, W. Cornell, P.A. Kollman, J. Phys. Chem. 97 (1993) 10269.

[51] M. Parrinello, A. Rahman, Phys. Rev. Lett. 45 (1980) 1196.

[52] W.G. Hoover, Phys. Rev. A 31 (1985) 1695

[53] W.G. Hoover, Phys. Rev. A 34 (1986) 2499.

[54] U. Essmann, L. Perera, M.L. Berkowitz, T. Darden, H. Lee, L.G. Pedersen, J. Chem. Phys. 103 (1995) 8577.

[55] M. Tuckerman, B.J. Berne, G.J. Martyna, J. Chem. Phys. 97 (1992) 1990.

[56] G. Ciccotti, J.P. Ryckaert, Comput. Phys. Rep. 4 (1986) 346.

[57] S. Marsili, G.F. Signorini, R. Chelli, M. Marchi, P. Procacci, J. Comput. Chem. 31 (2009) 1106.

[58] M.J. Frisch, G.W. Trucks, H.B. Schlegel, G.E. Scuseria, M.A. Robb, J.R. Cheeseman, G. Scalmani, V. Barone, B. Mennucci, G.A. Petersson, H. Nakatsuji, M. Caricato, X. Li, H.P. Hratchian, A.F. Izmaylov, J. Bloino, G. Zheng, J.L. Sonnenberg, M. Hada, M. Ehara, K. Toyota, R. Fukuda, J. Hasegawa, M. Ishida, T. Nakajima, Y. Honda, O. Kitao, H. Nakai, T. Vreven, J.A. Montgomery Jr., J.E. Peralta, F. Ogliaro, M. Bearpark, JJ. Heyd, E. Brothers, K.N. Kudin, V.N. Staroverov, R. Kobayashi, J. Normand, K. Raghavachari, A. Rendell, J.C. Burant, S.S. Iyengar, J. Tomasi, M. Cossi, N. Rega, J.M. Millam, M. Klene, J.E. Knox, J.B. Cross, V. Bakken, C. Adamo, J. Jaramillo, R. Gomperts, R.E. Stratmann, O. Yazyev, A.J. Austin, R. Cammi, C. Pomelli, J.W. Ochterski, R.L. Martin, K. Morokuma, V.G. Zakrzewski, G.A. Voth, P. Salvador, J.J. Dannenberg, S. Dapprich, A.D. Daniels, O. Farkas, J.B. Foresman, J.V. Ortiz, J. Cioslowski, D.J. Fox, Gaussian 09, Revision A.02., Gaussian Inc., Wallingford, CT, 2010.

[59] C. Bret, M.J. Field, L. Hemmingsen, Mol. Phys. 98 (2000) 751.

[60] H.A. Stern, F. Rittner, B.J. Berne, R.A. Friesner, J. Chem. Phys. 115 (2001) 2237.

[61] M.W. Mahoney, W.L. Jorgensen, J. Chem. Phys. 112 (2000) 8910.

[62] F.H. Stillinger, A. Rahman, J. Chem. Phys. 60 (1974) 1528.

[63] S.W. Rick, J. Chem. Phys. 120 (2004) 6085.

[64] B.P.F.W.H. Press, S.A. Teukolsky, W.T. Vetterling, Numerical Recipes in Fortran 77: The Art of Scientific Computing, 2nd ed. Cambridge University Press, New York, 1992.

[65] Y. Ouyang, F. Ye, Y. Liang, Phys. Chem. Chem. Phys. 11 (2009) 6082.

[66] S.L. Njo, J. Fan, B. Van De Graaf, J. Mol. Catal. A Chem. 134 (1998) 79

[67] P. van Genechten, K.A. Mortier, W.J. Geerlings, J. Chem. Phys. 86 (5063) (1987).

[68] E. Guàrdia, J. Martí, L. García-Tarrés, D. Laria, J. Mol. Liq. 117 (2005) 63.

[69] A.V. Guàrdia, E. Martí, J. Padró, J.A. Saiz, L. Komolkin, J. Mol. Liq. 96 (3) (2002).

[70] A. Luzar, D. Chandler, J. Chem. Phys. 98 (1993) 8160.

[71] L. Saiz, J.A. Padró, E. Guàrdia, J. Phys. Chem. B 101 (1997) 78.

[72] G. Pálinkás, E. Hawlicka, K. Heinzinger, Molecular dynamics simulations of watermethanol mixtures, Chem. Phys. 158 (1991) 65, https://doi.org/10.1016/03010104(91)87055-Z.

[73] K. Mizuno, Y. Miyashita, Y. Shindo, H. Ogawa, J. Phys. Chem. 99 (1995) 3225.

[74] A. Laaksonen, P.G. Kusalik, I.M. Svishchev, J. Phys. Chem. A 101 (1997) 5910.

[75] I. Bakó, T. Megyes, S. Bálint, T. Grósz, V. Chihaia, Phys. Chem. Chem. Phys. 10 (2008) 5004.

[76] M.D. Elola, B.M. Ladanyi, J. Chem. Phys. 122 (2005) 224506.

[77] M.T. Sonoda, S.M. Vechi, M.S. Skaf, Phys. Chem. Chem. Phys. 7 (2005) 1176.

[78] S. Sudo, N. Shinyashiki, Y. Kitsuki, S. Yagihara, J. Phys. Chem. A 106 (2002) 458.

[79] R. Ludwig, Chem. Phys. 195 (1995) 329.

[80] G. Guevara-Carrion, J. Vrabec, H. Hasse, J. Chem. Phys. 134 (2011), 074508,

[81] G. D’Arrigo, A. Paparelli, J. Chem. Phys. 88 (1988) 405.

[82] G. D’Arrigo, A. Paparelli, J. Chem. Phys. 88 (1988) 7687.

[83] A. Asenbaum, C. Pruner, E. Wilhelm, M. Mijakovic, L. Zoranic, F. Sokolic, B. Kezic, A. Perera, Vib. Spectrosc. 60 (2012) 102.

[84] R. Shuker, R.W. Gammon, Phys. Rev. Lett. 25 (1970) 222.

[85] K. Mizoguchi, Y. Hori, Y. Tominaga, J. Chem. Phys. 97 (1992) 1961.

[86] O.F. Nielsen, Chem. Phys. Lett. 60 (1979) 515.

[87] G.E. Walrafen, M.R. Fisher, M.S. Hokmabadi, W.H. Yang, J. Chem. Phys. 85 (1986) 6970.

[88] F. Paesani, W. Zhang, D.A. Case, T.E. Cheatham, G.A. Voth, J. Chem. Phys. 125 (2006) 184507.

[89] S. Sastry, H.E. Stanley, F. Sciortino, J. Chem. Phys. 100 (1994) 5361.

[90] G.E. Walrafen, Y.C. Chu, G.J. Piermarini, J. Phys. Chem. 100 (1996) 10363.

[91] M.D. Elola, B.M. Ladanyi, J. Chem. Phys. 126 (2007), 084504, .

[92] P.A. Maden, R.W. Impey, Phys. Lett. 123 (1986) 502.

[93] V. Mazzacurati, M.A. Ricci, G. Ruocco, M. Sampoli, Chem. Phys. Lett. 159 (1989) 383. 
94] G. Corongiu, E. Clementi, J. Chem. Phys. 98 (1993) 4984

[95] S. Burikov, T. Dolenko, S. Patsaeva, Y. Starokurov, V. Yuzhakov, Mol. Phys. 108 (2010) 2427

96] K. Egashira, N. Nishi, J. Phys. Chem. B 102 (1998) 4054.

[97] Y. Amo, Y. Tominaga, Chem. Phys. Lett. 320 (2000) 703.
[98] T. Fukasawa, Y. Amo, Y. Tominaga, J. Chem. Phys. 118 (2003) 6387.

[99] Y. Amo, Y. Inadachi, Y. Tominaga, J. Chem. Phys. 119 (2003) 10801.

[100] S. Xu, D. Bruce Chase, J.F. Rabolt, I. Noda, Appl. Spectrosc. 73 (2019) 1012 